\title{
ARTIGO
}

\section{Tratamento da Doença de Parkinson}

\author{
Henrique Ballalai Ferraz*
}

\begin{abstract}
RESUMO
A levodopa ainda é a pedra angular do tratamento da doença de Parkinson apesar de seu uso por longo prazo nảo ser isento de problemas. Nas fases oligossintomáticas podemos optar pela utilizaçāo de selegilina, anticolinérgicos e amantadina. Quando os sintomas săo um pouco mais proeminentes, mas nāo incapacitantes, os agonistas dopaminérgicos (DA) isoladamente são ùteis. Se hả algum grau de incapacidade nas atividades do dia-a-dia, ou se houver intoleráncia aos outros medicamentos. a levodopa deve ser iniciada. Esta deve ser mantida na menor dose possivel e se doses maiores forem requeridas, o melhor é adicionar agonistas DA. Na fase de flutuaçōes e discinesias, recomendamos como as principais medidas a serem tomadas a orientação dietética, o fracionamento das doses $\theta$ o uso de agonistas DA. O tratamento cirúrgico (talamotomia e palidotomia) tem um papel importante nas fases em que as complicações não são resolvidas com as medidas habituais.
\end{abstract}

\section{UNITERMOS}

Doença de Parkinson, levodopa, complicações motoras.
Médico Doutor em Neurologia e Chefe do Setor de Distúrbios do Movimento. Disciplina de Neurologia da Escola Paulista de Medicina - UNIFESP
A doença de Parkinson (DP) é uma moléstia de curso progressivo, geralmente com início assimétrico em uma metade do corpo. Pode manifestar-se combinando dois ou mais dos seguintes sinais: tremor de repouso, rigidez muscular, acinesia c compromelimento dos reflexos de manutenção de postura.

As manifestaçōes clínicas da doença decorrem de uma deficiência de dopamina (DA) cerebral resultante de degeneração dos neurônios pigmentados da substância negra mesencefálica. A causa da DP ainda não é conhecida até o presente, mas há evidências de que fatores genéticos, ambientais e o envelhecimento podem estar envolvidos na etiopatogênese.

Há várias formas de tratamento para a doença, mas a medida mais eficaz consiste em restabelecer, ao menos parcialmente, a transmissão dopaminérgica. O uso da levodopa é uma das formas para se restaurar essa neurotransmissão e, no presente, ainda é a mais efetiva e viável de todas'. A levodopa penetra no sistema nervoso central e, por ação da enzima dopa descarboxilase cerebral. é convertida em dopamina?.

O surgimento da levodopa revolucionou o tratamento da DP no final da década de 60. Pacientes que estavam seriamente comprometidos, muitos confinados à cama ou à cadeira-de-rodas, recuperaram a mobilidade com a nova droga. A DP tornou-se a primeira doença degenerativa do sistema nervoso a ser tratada com a reposição de neurotransmissores. Apesar do impacto inicial positivo, alguns problemas surgiram com a levodopa. A intolerância gastrintestinal foi um dos efeitos colaterais mais observados no início e, além disso, alterações psiquiátricas e hipotensão ortostática foram notadas em alguns pacientes. Essas complicações tornaram-se mais raras quando os inibidores da dopa descarboxilase periférica foram incorporados aos comprimidos de levodopa. Passado algum tempo, outros problemas surgiram com o tratamento prolongado, em particular as flutuações do rendimento motor e as discinesias induzidas pela levodopa. Hoje sabemos que cerca de metade dos pacientes, após cinco anos de tratamento com a levodopa, vai apresentar essas complicações'. Apesar disso $\mathrm{e}$ do surgimento de novas alternativas terapêuticas, a levodopa permanece até hoje como a droga mais eficaz no tratamento da DP.

Até há bem pouco tempo, suspeitava-se que as flutuações c discinesias associadas ao uso da levodopa eram decorrentes do tempo de uso da droga 
e, por esta razão, havia uma tendência em reservar-se o uso da levodopa para os pacientes com formas mais avançadas da doença ou que, pelo menos, manifestassem comprometimento funcional nas atividades do dia-a-dia. Mais recentemente, tem sido demonstrado que a levodopa em si não parece ser tóxica para as células do SNC e muitas evidências apontam para que o tempo decorrido de doença é que é o fator decisivo para produzir modificações nos circuitos neurais, favorecendo o aparecimento das flutuações e discinesias'.

Com relação às outras drogas antiparkinsonianas, sabemos que as flutuações não acontecem e as discinesias também são incomuns, mesmo usando-as por um período prolongado. Apesar do uso da levodopa estar cada vez mais liberalizado, podemos optar por não utilizá-la nos pacientes com formas brandas e oligossintomáticas da DP.3.

\section{OPÇÃO PELA NÃO-UTILIZAÇÃO DA LEVODOPA}

Se a opção é pela não-utilização da levodopa, temos disponível para uso inicial uma das seguintes drogas, isoladamente ou em combinação: selegilina, anticolinérgicos (biperideno ou triexifenidila), amantadina e agonistas dopaminérgicos (bromocriptina, pergolida, pramipexol).

A selegilina é uma droga que atua inibindo irreversivelmente a enzima monoaminoxidase $\mathrm{B}$ (MAO-B) que é uma enzima com distribuição generalizada no cérebro. No metabolismo da DA cerebral, esta, depois de liberada na sinapse, é recaptada pelo neurônio pré-sináptico. Dentro do neurônio e das células da glia a MAO-B degrada a dopamina. Enquanto a MAO-B parece atuar especificamente sobre a dopamina, a MAO-A preferentemente atua sobre a tiramina, serotonina $(5-\mathrm{HT})$ e norepinefrina (NE). Essa especificidade da MAO-B permite que a utilização da selegilina não esteja associada ao cfcito hipertensor e taquicárdico (cheese effect) que costuma ser observado com a utilização dos inibidores não-seletivos da MAO. O aumento da disponibilidade da dopamina dentro das células seria um dos possíveis mecanismos de ação da droga. Outro mecanismo plausível para a ação da selegilina seria sua metabolização em derivados anfetamínicos que podem ter alguma alividade antiparkinsoniana decorrente do efeito estimulante sobre o SNC (explicando também a insônia, um de seus cleitos colaterais $)^{3}$.

A possibilidade de haver um efeito ncuroprotetor surgiu a partir da demonstração de que a selegilina impede a produção de parkinsonismo nos animais de experimenıação submetidos à exposição do MPTP (metil-fenil-tetra-hidro-piridina). O MPTP é uma protoxina que sob a ação da MAO-B da glia convertesc cm MPP+, a toxina que de fato consegue penetrar no neurônio dopaminérgico e destruí-lo. Não sabemos cxatamente como ocorre a destruição do neurônio na DP, mas se algum mecanismo tóxico houver com a parlicipação da MAO, a selegilina poderia ter um papel preventivo. Outro possível papel neuroprotetor reside no fato de que a inibição da MAO resultaria numa diminuição do processo oxidativo intracelular e portanto diminuiria a produção de radicais livres. Um estudo cooperativo com um grande número de pacientes realizado na América do Norte (DATATOP) demonstrou que a utilização de selegilina nas fases iniciais da DP é capaz de retardar a necessidade de levodopa, o que, em outras palavras, significa reduzir o ritmo de progressão dos sintomas ${ }^{4}$. Algumas evidências apontam para que essa ação da selegilina ocorra pelo seu efeito sintomático e não por uma neuroproteção verdadeira. Uma delas basciil-se no lato de que esse retardo na progressão não se mantém depois de dois anos e, outra, que a selegilina não previne o aparecimento das discinesias e flutuações ${ }^{1}$. Além disso, o efcito antioxidante da selegilina só é observado in vitro.

De qualquer modo, haja ou não um efeito neuroprolctor, a selegilina é uma boa opção para aqueles pacientes cujos sintomas são leves e não produzam nenhum tipo de incômodo significativo. Tem como efeito colateral mais frequiente a insônia e, por isso, deve-se evitar a tomada da droga no período vespertino ou noturno.

Há muito tempo os anticolinérgicos vêm sendo utilizados empiricamente na DP e sua atuaçũo hoje pode ser explicada pela preponderância de acetilcolina observada no estriado dos pacientes com diminuição da neurotransmissão dopaminérgica a partir da substância negra. Essas drogas são razoavelmente eficazes sobre o tremor c a rigidez muscular, mas a atuação sobre a acinesia, que é o sintoma mais debilitante da DP, é desprezível. Reservamos os anticolinérgicos para aqueles pacientes com formas unilaterais ou predominantemente assimétricas e nos quais a acinesia não ć significativa. $O$ uso é limitado pelo aparecimento de efeitos colaterais periféricos (boca seca, obstipação, retenção urinária, turvação visual) e centrais (sonolência, confusão mental, delírios e alucinaçōes) que são muito mais frequientes a partir dos 65 anos e nos pacientes com algum grau de declínio cognitivo. É importante salientar que os anticolinérgicos são contra-indicados nos pacientes com glaucoma ou com prostatismo. 
A amantadina ć uma outra opção no tratamento dos pacientes nas fases iniciais da DP. Seu mecanismo de ação não está totalmente esclarecido. mas há evidências de que aumente a liberação de DA para a fenda sináptica. Sabe-se que também apresenta um fraco efeito anticolinérgico $\mathrm{c}$ mais recentemente foi demonstrado exercer uma ação bloqueadora de receptores $\mathrm{N}$-metilD-aspartato (NMDA) ${ }^{3}$. O bloqueio dos receptores NMDA é capaz de facilitar a transmissão DA no estriado. A amantadina atua razoavelmente bem na rigidez, tremor e acinesia. Alguns pacientes podem experimentar uma perda da eficácia após algum tempo de tratamento, mas a suspensão do uso por 30 dias é capaz de fazer reverter a situação. Tem como efeitos colaterais mais comuns o aparecimento de livedo reticular e edema de tornozelo. Alguns pacientes podem queixar-se de efeitos anticolinérgicos.

Os agonistas DA, ao contrário da selegilina, anticolinérgicos e amantadina, atuam dirctamente nos receptores da dopamina, não necessitando passar por uma metabolização no neurônio pré-sináptico. Há duas famílias de receptores DA: os receptores D1, estimuladores da enzima adenil ciclase, e os receptores D2, indutores de inibição da adenil ciclase. Os agonistas mais utilizados são a bromocriptina, a pergolida $\mathrm{c} o$ pramipexol ${ }^{5}$. Teoricamente, os agonistas são drogas mais potentes que os anticolinérgicos e a amantadina (mas menos que a levodopa) e costumam ser reservados para aqueles pacientes um pouco mais sintomáticos. Da mesma forma que a selegilina, o uso dos agonistas DA permite que a introdução da levodopa seja postergada, c com a vantagem de não induzir flutuações do desempenho motor ${ }^{-4}$. São drogas mais caras e devem ser iniciadas em doses bem baixas, pois, do contrário, os pacientes desenvolvem acentuada intolerância à medicação, manifestando-se com náuseas, vômitos, hipotensão ortostática, confusão mental, alucinações e delírios.

Algumas vezes, utilizamos as drogas mencionadas em combinação de duas ou até três, embora a necessidade de combinar duas ou mais dessas drogas seja um forte indício de que o paciente esteja precisando receber a levodopa. O quadro 1 mostra as linhas gerais que seguimos na opção de não utilizar a levodopa.

\section{OPÇÃO PELA UTILIZAÇÃO DA LEVODOPA}

Quando o controle dos sintomas parkinsonianos já não ś mais satisfatório com as drogas anteriormente citadas, é o momento de utilizar a levodopa.

O mecanismo de ação básico da levodopa está centrado na capacidade de esta ser convertida em dopamina dentro do cérebro e corrigir o estado de deficiência DA característico do parkinsonismo². Apesar disso, mesmo após mais de 25 anos de uso bem-sucedido da levodopa na doença de Parkinson, não sabemos exalamente como ocorre essa conversão no estriado. A hipótese clássica do mecanismo de ação central supõe que os terminais sinápticos nigroestriatais sobreviventes dos parkinsonianos (sabe-se que na época do início dos sintomas da DP. cerca de $50-60 \%$ dos neurônios DA já foram degenerados) captam a levodopa e a bateria enzimática do neurônio encarrega-se de converter a DA, estocá-la $\mathrm{em}$ vesículas e liberá-la para a fenda sináptica. Existem hipóteses alternativas, todas elas com cmbasamento científico. Uma delas advoga que a DA

\section{QUADRO 1}

Linhas gerais de utilização de antiparkinsonianos não-associados à levodopa

- Selegilina (Deprilan, Jumexil, Niar): iniciar com 2,5 a $5 \mathrm{mg}$ ao dia e manutenção com 2,5 a $10 \mathrm{mg}$ ao dia ( $1-2 \times /$ dia)

- Triexifenidila (Artane): iniciar com $1 \mathrm{mg} / \mathrm{dia}$ e manter com doses entre $4 \mathrm{e} 15 \mathrm{mg}$ ao dia (2-3x/dia)

- Biperideno (Akineton): iniciar com $1 \mathrm{mg} / \mathrm{dia}$ e manter com doses entre 4 e $12 \mathrm{mg}$ ao dia (2-3x/dia)

- Bromocriptina (Parlodel, Bagren): iniciar com $2,5 \mathrm{mg} /$ noite e incrementar a dose semanalmente até atingir o efeito desejado (dose útil entre 7,5-30 mg/dia. dividida $3 \mathrm{x} / \mathrm{dia}$ )

- Pergolida (Celance): iniciar com $0,05 \mathrm{mg} / \mathrm{noite}$ e incrementar a dose semanalmente até obter o efeito desejado (dose útil entre 0,5-3 $\mathrm{mg} / \mathrm{dia}$, dividida $3 \times /$ dia)

- Pramipexol (Mirapex. Sifrol): iniciar com $0.125 \mathrm{mg} /$ dia e incrementar a dose semanalmente até obter o efeito desejado (dose útil entre $1.5-4,5 \mathrm{mg} / \mathrm{dia}$, dividida em $3 \mathrm{x} / \mathrm{dia}$ ) 
convertida a partir da levodopa exógena não fica estocada em vesículas, mas livre no citoplasma neuronal. Outras evidências apontam para o fato de que a maior parte da levodopa administrada oralmente é convertida em dopamina em outros neurônios não-dopaminérgicos do cérebro ou que esta conversão ocorra nas células da glia adjacentes aos receptores DA estriatais².

A levodopa é rapidamente absorvida no duodeno e jejuno proximal e tem uma meia vida plasmática variando de 50 a 120 minutos. Perifericamente é descarboxilada pela dopa descarboxilase (convertida em dopamina) e pela catecol-O-metil-transferase - COMT (convertida em 3-O-metil-dopa). É transportada para dentro do cérebro pelos mesmos sistemas de transporte ativo utilizados por outros aminoácidos de cadeia leve?

A levodopa nas formulações comerciais disponíveis vem associada a inibidores da dopa descarboxilase periférica para impedir a conversão em dopamina na corrente sangüínea. No Brasil temos disponíveis a levodopa associada à benserazida (Prolopa) e à carbidopa (Sinemet). Há apresentações que permitem uma liberação gradual da droga no tubo digestivo com uma duração maior do seu efeito (Prolopa HBS e Cronomet).

Deve-se iniciar a levodopa gradualmente e administrá-la longe das refeições para otimizar a absorção. Alguns pacientes desenvolvem uma extrema intolerância à droga, mesmo em doses mínimas, com náuseas, vômitos, sudorese, hipotensão ortostática e alucinações. Às vezes as náuseas e vômitos são incontroláveis mesmo administrando a lcvodopa com a alimentação e associando-se anticméticos, como o domperidone e a cisaprida, o que acaba por impedir sua utilização. A metoclopramida deve ser evitada nos pacientes com DP uma vez que ela penetra no cérebro, no qual exerce uma ação antidopaminérgica.

A levodopa pode ser a escolha para iniciar o tratamento da DP desde que o médico julgue necessário. Caso o paciente esteja recebendo tratamento com outros antiparkinsonianos e a doença evolua para uma situação em que a levodopa seja necessária, esta pode ser adicionada ao esquema posológico já utilizado sem modificar o restante da medicação.

O uso de levodopa concomitante com a selegilina é controverso. O Grupo de Estudo em Parkinson do Reino Unido encontrou, em 520 pacientes ao longo de 5 anos de acompanhamento, uma mortalidade aumentada no grupo de pacientes recebendo as drogas combinadamente quando comparada aos que recebiam apenas levodopa (mortalidade de $28 \%$ vs. $17,7 \%)^{6}$. Esse estudo foi criticado por problemas metodológicos e, além disso, outros grupos não tiveram a mesma experiência. Até que essa questão esteja bem esclarecida, há quem prefira não usar associadamente as duas drogas.

O esquema que utilizamos para introduzir a levodopa está colocado no quadro 2.

O ideal é manter a menor dose possível de levodopa c, no caso do paciente responder a esta droga, mas necessitar de doses além daquelas sugeridas no quadro 2, os agonistas DA podem ser agregados ao tratamento, no mesmo csquema recomendado no quadro 1 . Um pequeno número de pacientes não tem qualquer resposta a doses baixas de levodopa e, neste caso, são mantidos, mesmo em lases não avançadas, com doses tão altas quanto $1.500 \mathrm{mg}$ ao dia (6-7 comp/dia).

O quadro 3 apresenta algumas medidas úteis para o clínico levar em consideração em qualquer fase do tratamento da DP.

\section{MANEJO DA FASE MAIS AVANÇADA DA DOENÇA DE PARKINSON}

No decorrer da fase mais tardia da DP, freqüentemente são necessários ajustes na dosagem da levodopa e dos agonistas DA, uma vez que o processo degenerativo das células nigroestriais segue seu ritmo. As flutuaçóes do rendimento motor da levodopa e as

\section{QUADRO 2}

\section{Esquema para utilização de levodopa}

- Prolopa 250 (levodopa 200 + benserazida 50): iniciar com $1 / 4$ a $1 / 2$ comp $2 x /$ dia e aumentar até o efeito desejado. Ideal manter com doses abaixo de 2 comp/dia (em 3-4 tomadas)

- Prolopa HBS (levodopa 100 + benserazida 25): iniciar com 1 cáp/dia. Ideal manter abaixo de 4 cáp/dia (em 2-3 tomadas)

- Cronomet (levodopa $200+$ carbidopa 25): iniciar com 1 comp $1 \mathrm{x} /$ dia. Ideal manter com doses abaixo de 2 ou 3 comp/dia (2-3 tomadas)

- Sinemet (levodopa $250+$ carbidopa 25 ): iniciar com $1 / 2$ comp $2 x /$ dia. Ideal manter abaixo de 2 comp/dia (3-4 tomadas) 


\section{QUADRO 3}

\section{Medidas sensatas no tratamento da doença de Parkinson}

- Iniciar uma droga de cada vez e só associar outra droga depois da primeira estar estabilizada e na dose desejada

- Não associar droga antidopaminérgica (metoclopramida, neurolépticos, flunarizina e cinarizina, entre outras) ao tratamento

- Iniciar drogas em doses pequenas e aumentá-las gradualmente até o palamar desejado (especialmente a levodopa, os agonistas DA $\mathrm{c}$ os anticolinérgicos)

- Não prescrever anticolinérgicos a pacientes com mais de 65 anos, com glaucoma, prostatismo ou com algum grau de demência

- Concluir pela ineficácia da levodopa apenas depois de atingir a dose de $1.500 \mathrm{mg}$ ao dia (cerca de 6 a 7 comprimidos dos preparados comerciais com inibidor da dopa descarbuxilase)

- Adicionar agonista dopaminérgico (bromocriptina, pergolida, pramipexol) aos pacientes que necessitarem doses elevadas de levodopa para permitir uma posterior redução destas doses

discinesias são as causas mais comuns de insucesso do tratamento nas fases avançadas da DP. Os tipos de flutuação motora mais comuns são a "deterioração de final de dose" (wearing-off) e as flutuações aleatórias, também conhecidas como "fenômeno on-off".

\section{Deterioração de final de dose (wearing-off)}

Caracteriza-se por um encurtamento da duração do efeito motor da levodopa, fazendo com que o paciente tenha o benefício da medicação ("estado on") por 2 ou 3 horas, necessitando receber uma nova dose para voltar à mobilidade. Em alguns pacientes, à medida que a doença progride, o "estado on" pode ser tão curto quanto $30 \mathrm{ou}$ 40 minutos. Quando o wearing-off instala-se, a tendência natural em acrescentar novas doses de levodopa não é a melhor medida, pois cada vez mais novos acréscimos terão que ser feitos com o tempo, fazendo com que o indivíduo venha a tomar uma dosagem inviável de levodopa em pouco tempo. Inicialmente recomenda-se checar se o paciente está recebendo a levodopa longe das refeições e orientar para que diminua o consumo de alimentos protéicos (para impedir a competição com aminoácidos da diela) no período útil do dia ${ }^{3}$. O máximo fracionamento possível das doses de levodopa, de preferência sem aumentar a dose total, é útil nessa situação. Os pacientes que ainda não estão recebendo agonista DA se beneficiarão com a introdução de uma das drogas do grupo. Temos uma maior experiência com a bromocriptina c com o pramipexol nessa fase e, em nossa opinião as duas medicações equivalem-se em eficácia mas a segunda é melhor tolerada pelos pacientes ${ }^{7}$.
A utilização de preparados de liberação lenta da levodopa (Prolopa HBS e Cronomet) permite uma maior duração do efeito e pode substituir as formulações standard (Prolopa c Sinemet) nos pacientes flutuadores. Os preparados líquidos de levodopa deverão estar disponíveis para uso clínico em pouco tempo e permitirão a administração de doses pequenas em curtos intervalos de tempo. Recentemente, o surgimento de drogas inibidoras da COMT - o tolcapone foi a primeira delas com este mecanismo de ação - abriu um novo horizonte para os pacientes com flutuação do rendimento da levodopa ${ }^{3}$. A despeito de ter mostrado eficácia clínica, com pouco tempo de uso, o relato isolado de casos de hepatopatia grave e fatal em 3 pacientes entre todos em uso da droga no mundo (cerca de 150.000 pacientes) fez com que seu uso tivesse de ser restrito alos casos mais graves. Ainda assim, se o tolcapone for prescrito, o paciente deve fazer controle de enzimas hepáticas a cada 15 dias e suspender a droga se as enzimas aumentarem. O entacapone é um outro inibidor da COMT em vias de ser lançado comercialmente com a vantagem de ter o mesmo perfil de eficácia clínica do tolcapone, sem, possivelmente, seus cfeitos adversos.

\section{Fenômeno on-off}

Também conhecido como efeito ioiô. Nesse fenômeno ocorre uma mudança brusca do estado de mobilidade do paciente sem que haja uma relação com o horário de tomada das doses da levodopa. Alguns pacientes podem ficar horas em estado off, ou seja, completamente acinéticos, a despeito de tomarem sucessivas doses da levodopa. Por serem imprevisíveis, essas flutuações são extremamente incapacitantes. Esse 
tipo de complicação é o mais difícil de manejar. As medidas sugeridas para o manejo das flutuações previsíveis (wearing-off) podem ajudar, mas na maioria das vezes são insuficientes para minorar a situação. $\mathrm{O}$ uso do agonista DA, apomorfina, é uma saída para os indivíduos com acinesia prolongada ${ }^{8}$. A apomorfina é uma droga potente com a desvantagem de ter de ser administrada por via parenteral e por apresentar um potente efeito indutor de náuseas e vômitos. O domperidone administrado em conjunto com a apomorfina permite um controle satisfatório do efeito emético. A apomorfina pode ser administrada por via subcutânea, pelo próprio paciente, na dose de $1-2 \mathrm{mg}$ (o equivalente a $0,1-0,2 \mathrm{~mL}$ dos preparados comerciais), levando a uma reversão do estado off em menos de 10 minutos e com duração do efeito entre 60-80 minutos, podendo ser repetida a cada 3 horas, se necessário ${ }^{8}$. No Brasil, a apomorfina pode ser obtida de importadores de medicamentos.

\section{Discinesia}

As discinesias são divididas em dois grandes grupos: as do período on e as do período off. As discinesias de período on são, na maioria das vezes, movimentos coreoatetóticos nas extremidades e segmento cranial, podendo manifestar-se apenas no auge do efeito da levodopa (discinesia-de-pico-de-dose) ou durante todo o efeito motor (discinesia-em-onda-quadrada ${ }^{9}$. Para obterse alguma melhora, temos de diminuir a dosagem da levodopa. Para que isso possa acontecer sem que provoque um aumento nos períodos off temos de adicionar agonista DA ao tratamento, ou aumentar sua dosagem, caso este já venha sendo usado. $O$ uso da amantadina, em função de sua ação antagonista de receptor NMDA, também tem sido recomendado nesses casos.

As discinesias de período off em geral são caraclerizadas por movimentos distônicos na região axial e lambém nas extremidades, e não raro são acompanhadas de dor no segmento acometido. Podem aparecer também no período matinal, despertando o paciente com dor. As medidas tomadas para controlar os períodos off podem ajudar $\mathfrak{e}$, quando estas não são suficientes, é necessário o acréscimo de dose extra de levodopa nos períodos da discinesia ${ }^{3}$.

\section{TRATAMENTO NÃO-MEDICAMENTOSO}

A fisioterapia e a terapia ocupacional têm um papel importante no tratamento da DP, especialmente naqueles pacientes com distúrbio acentuado do equilíbrio e da marcha. A diminuição do volume vocal e a disartria são distúrbios freqüentes e pouco responsivos ao tratamento medicamentoso. A fonoterapia tem um papel decisivo nesses pacientes ${ }^{10}$.

Nos últimos cinco anos assistimos a uma explosão do interesse no tratamento cirúrgico da fase avançada da DP. Que é uma alternativa útil para uma parcela dos pacientes com complicações motoras graves e em qual já foram tentadas todas as alternativas farmacológicas disponíveis, não se tem mais dúvidas. $O$ ponto central do debate atual reside em duas questões: qual o melhor alvo a ser escolhido para realizar a intervenção (talamotomia ou palidotomia) e qual o melhor procedimento (lesão ou estimulação).

Tanto a talamotomia quanto a palidotomia têm o seu papel no tratamento. A talamotomia é melhor indicada naqueles pacientes com predomínio de tremor e em formas unilaterais ou dominantemente assimétricas e que não toleram nenhum dos medicamentos disponíveis ". Vale ressaltar que este é um contingente muito pequeno de pacientes, já que essas formas habitualmente respondem bem ao tratamento medicamentoso e à intolerância, na maioria das vezes, pode ser contornada com as medidas habituais.

A palidotomia está indicada nas formas complicadas com flutuações e discinesias que não foram satisfatoriamente controladas com as medidas já anteriormente mencionadas. A resposta neste grupo de pacientes às vezes é surpreendente, havendo muitas vezes, além da melhora das flutuações e da discinesia, uma redução nas doses dos antiparkinsonianos ${ }^{11.12}$. O efeito dos dois tipos de cirurgia é notado logo no pós-operatório e pode durar por dois anos ou mais ${ }^{12}$.

A cstimulação elétrica dos núcleos da base (especialmente do núcleo subtalâmico) com o implante de eleIrodos é uma alternativa ao tratamento cirúrgico lesivo, tendo a vantagem de permitir uma suspensão do tratamento caso se deseje e de permitir que se faça o procedimento bilateral, sem o risco de haver disartrofonia grave ${ }^{13}$. As desvantagens são o custo elevado do procedimento, risco de infecção e falhas no equipamento de estimulação. No nosso meio, a maior experiência é com os procedimentos ablativos, que, feitos por uma equipe experiente, têm um baixo risco de complicações ou seqüiclas definitivas.

O tratamento cirúrgico, como o implante no estriado de células DA da substância negra fetal ou do transplante autólogo de células DA do corpo carotídeo, é uma perspectiva promissora para os próximos anos.

\section{SUMMARY}

Parkinson's Disease Management

Levodopa remains the mainstay in Parkinson's disease treatment, although long-term problems may emerge with continuous use. In early symploms, 
selegiline, anticholinergic drugs and amantadine are preferred. When symptoms are more proeminent but not incapacitating, dopaminergic agonists can be prescribed. Levodopa therapy is indicated when other drugs no longer provide satisfactory control of symptoms or are not tolerated by the patient. Adding dopaminergic agonists to treatment is a wise measure if higher dosis of levodopa is required. Dietetic orientation, fractioning of the dosis and dopaminergic agonists are necessary in fluctuating and dyskinetic patients. Surgical approach, such as thalamotomy and pallidotomy, are only indicated if non-invasive measures were ineffective.

\section{KEY WORDS}

Parkinson's disease, levodopa, motor complications.

\section{Referências}

1. Lang AE \& Lozano AM. Medical progress: Parkinson's disease. Part 2. N Engl J Med, 339: 1144-1153, 1998.

2. Poewe W, Wenning G. Levodopa in Parkinson's disease: mechanisms of action and pathophysiology of late failure. In: Jankovic J, Tolosa $\mathrm{E}$ (eds). Parkinson's disease and movement disorders. Baltimore, William \& Wilkins, 177-190, 1998.

3. Jankovic J, Marsden CD. Therapeutic strategies in Parkinson's disease. In: Jankovic J, Tolosa E (eds). Parkinson's disease and movement disorders. Baltimore, William \& Wilkins, 191220, 1998

4. Parkinson Study Group. Effects of tocopherol and deprenyl on the progression of disability in early Parkinson's disease. N Engl J Med, 328: 176-183, 1993.

5. Shannon KM, Bennet Jr JP, Friedman JH. Efficacy of pramipexole, a novel dopamine agonist, as monotherapy in mild to moderate Parkinson's disease. Neurology, 49: 724$728,1997$.

6. Lees AJ, Head J, Bem-Schlomo Y. Selegiline and mortality in Parkinson's disease: another view. Ann Neurol, 41: 282-283, 1997.
7. Ferraz HB, Azevedo-Silva SMC, Borges $V$ et al. Apomorfina. Uma alternativa no controle das flutuações motoras da doença de Parkinson. Arq Neuropsiquiatr, 53: 245-251, 1995.

8. Lieberman A, Ranhosky A, Korts D. Clinical evaluation of pramipexole in advanced Parkinson's disease: results of a double-blind, placebo-controlled, parallel-group study. Neurology, 49: 162-168, 1997.

9. Rocha MSG, Andrade LAF, Ferraz HB, Borges V. Discinesias induzidas por levodopa em 176 pacientes com doença de Parkinson. Arq Neuropsiquiatr, 53: 737-742, 1995.

10. De Angelis EC, Mourāo LF, Ferraz $\mathrm{HB}$ et al. Effect of voice rehabilitation on oral communication of Parkinson's disease patients. Acta Neurol Scand, 96: 199-205, 1997.

11. Ferraz FP, Aguiar PMC, Ferraz $\mathrm{HB}$ et al. Talamotomia e palidotomia estereotáxica com planejamento computadorizado no tratamento da doença de Parkinson. Avaliação do desempenho motor a curto prazo de 50 pacientes. Arq Neuropsiquiatr, 56: 789-797, 1998.

12. Lang $A E$, Lozano A, Montgomery $E$ et al. Posteroventral medial pallidotomy in advanced Parkinson's disease. N Engl J Med, 337: 1036-1042, 1997.

13. Limousin $P$, Krack $P$, Pollak $P$ et al. Electrical stimulation of the subthalamic nucleus in advanced Parkinson's disease. $N$ Engl J Med, 339: 1105-1109, 1998.

Endereço para correspondēncia

Dr. Henrique B. Ferraz

Disciplina de Neurologia

Rua Botucatu, 740

CEP 04023-900 São Paulo (SP)

E-mail: henrique@sun-nepi.epm.br

ANAFRANIL- - Formas farmacéuticas e apresentaçōes: Drágeas com 10 ou $25 \mathrm{mg}$ de cloridrato de clomipramina; excipiente q.s.p. 1 drágea. Caixas com 20 drágeas. Ampolas com $25 \mathrm{mg}$ de cloridrato de clomipramina; veiculo q.s.p. $2 \mathrm{ml}$. Caixas com 10 ampolas. Anafranil SR: cada comprimido de liberação lenta contèm cloridrato de clomipramina $75 \mathrm{mg}$; excipiente q.s.p. 1 comprimido. Caixas com 20 comprimidos. Posologia: 0 esquema posológico deve ser estabelecido individualmente. A dose diárı usual e de $75-150 \mathrm{mg}$ (em ataques de pânico, $25-100 \mathrm{mg} /$ dia). Iniciar o tratamento com um comprimido de $25 \mathrm{mg}$, duas a três vezes ao dia. ou com um comprimido de liberação lenta de $75 \mathrm{mg}$, uma vez ao dia: em ataques de pánıco. iniciar com um comprimido de $10 \mathrm{mg}$ por dia. Evitar a interrupçảo abrupta do tratamento. Contra-indicações: Hipersensibilidade à clomipramina ou aos excipientes; sensibilidade cruzada aos antidepressivos triciclicos do grupo dibenzazepina. Infarto do miocárdio recente. Usó concomitante com inibidores da MAO. Precauçōes e advertèncias: Distürbios da conduçào cardiaca insuficiència cardiovascular. baixo limiar convulsivo, glaucoma de àngulo agudo, distúrbios da miç̧ão, insuficiência hepática grave, tumores da medula adrenal, terapia eletroconvulsiva. Hipertireoidismo ou tratamento concomitante com preparaçòes tireoidianas. Constipação crônica. Monitorizaçâo da contagem de células sangüineas e da função hepatıca. Intervenções cirúrgicas. Risco de suicidio. Choque anafilático (uso parenteral). Uso concomitante com drogas anti-hipertensivas. drogas simpatomiméticas, depressores do SNC, anticoliı érgicos. inibıdores seletivos da recaptação da serotonina, neurolépticos. indutores das enzimaş hepatica, anticoagulantes. cimetidina. metilfenidato e estrogenos. Nào deve ser usado durante a gravidez e a lactaçào. Cuidado ao ope-rar máquinas e dirigir veiculos. Reaçöes adversas: Frequentes: reaçóes no SNC e reaçóes antıcolinérgicas (sonolência, fadiga, tremor. mioclonia, aumento do apetite, boca seca. distürbios da mıç̧ao. distúrbios visuais). ganho de peso. Ocasionais: confusão. alucinaçōes, agitaçảo. distübios visuaıs, elevaçào das transaminases, reaçōes alérgicas cutâneas, distúrbios gastrintestinais. Raras: ativação de sintomas psicóticos. convulsōes, ataxia, arritmıas. hıpertensảo. Casos isolados: glaucoma. disturbios da conduçào cardiaca. hepatite. contagem anormal das rélulas sangüineas, alveolite alérgıca. reaçoes anafiláticasianafilactoides e hiperpirexia.

Intormaçòes completas para prescriçào à disposiçào da classe médica mediante solicitaçào. 